\title{
Review: few elderly inpatients who are depressed improve
}

Cole MG, Bellavance F. Depression in elderly medical inpatients: a meta-analysis of outcomes. CMAJ 1997 Oct 15;157:1055-60.

\section{Objective}

To determine the prognosis of medical inpatients who are elderly and have depression.

\section{Data sources}

Studies were identified by searching Medline (1980 to September 1996) and PsychINFO (1984 to September 1996) databases using the keywords depression, prognosis, course, follow up, and aged and by scanning the bibliographies of relevant papers.

\section{Study selection}

Studies were selected if they reported original research, were published in English or French, had a study population of medical inpatients whose mean age was $\geqslant 60$ years, and reported affective state as an outcome. Studies of patients with exclusively 1 condition were excluded.

\section{Data extraction}

Data were extracted by 1 reviewer on the methods and design of the studies (formation of an inception cohort, description of referral pattern, length and completion of follow up, objective outcome criteria, unbiased outcome assessment, and adjustment for extraneous prognostic factors); patient population; diagnostic criteria; proportion of cases detected and treated; affective outcomes; and prognostic factors.

\section{Main results}

8 studies involving 265 depressed patients met the inclusion criteria. All studies assembled inception cohorts and follow up was complete in 5 studies. All studies had some methodological flaws, particularly in the description of referral patterns, use of unbiased outcome assessments, length of follow up, and adjustment for extraneous prognostic factors. Follow up was $\leqslant 3$ months in 5 studies (mean $1.5 \mathrm{mo}$ ) and $\geqslant 12$ months (mean 18 mo) in 4 studies ( 1 study reported data for both follow up periods). 5 studies reported the rates of eventual antidepressant treatment (median 46\%, range rare to $100 \%$ ). 3 outcome categories were consistent across the studies: well, depressed, and dead. When the results for 4 studies that followed up patients for $\geqslant 12$ months were combined, $19 \%$ of patients were well (95\% CI $6 \%$ to $32 \%$ ), $29 \%$ were depressed (CI 9\% to 50\%), 53\% died (CI 18\% to $88 \%$, only 2 studies reported deaths), and $22 \%$ had other outcomes (CI 15\% to 29\%) such as dementia, partial remission, loss to follow up, and refusal. When the results for 5 studies that followed up patients for $\leqslant 3$ months were combined, $18 \%$ of patients were well (CI 6\% to 30\%), 43\% were depressed (CI 24\% to $61 \%$ ), $22 \%$ died (CI $0 \%$ to $49 \%$, only 3 studies reported deaths), and $22 \%$ of patients had other outcomes (CI $15 \%$ to $28 \%$ ). Heterogeneity existed across studies for 3 of the 4 outcomes. When 1 study in which all patients were treated and followed up was removed, heterogeneity existed for 1 of 4 outcomes.

\section{Conclusion}

Most studies on the prognosis of depression in inpatients who are elderly have flaws; these studies suggest that prognosis is relatively poor with $19 \%$ of patients becoming well.

Source of funding: no external funding.

For article reprint:Dr M G Cole, Division of Geriatric Psychiatry, St. Mary's Hospital Center, 3830 Lacombe Avenue, Montreal, Quebec H3T IM5, Canada.

Abstract and commentary also published in Evidence-Based Medicine $1998 \mathrm{Jul}$-Aug.

\section{Commentary}

Substantial depression is found in $15 \%$ to $20 \%$ of medical patients who are elderly, ${ }^{1}$ but there is little to guide the clinician on how best to treat these patients. They are a difficult group to research. The overlap of medical and psychiatric symptoms makes psychiatric assessment problematic, and attrition rates in such studies are usually about 50\%. Hence, Cole and Bellavance found only 8 studies which met reasonable inclusion criteria. Pooling this kind of data is bound to result in rather imprecise outcome figures. Nevertheless, the striking finding is that the outcome is poor-only 1 in 5 patients recovered. This is similar to community studies of people who are elderly and depressed, but considerably worse than patients treated by psychiatrists. Indeed, Cole's earlier meta-analysis of elderly patients with depression who were treated by psychiatrists showed a far more favourable outcome; $60 \%$ of patients recovered. ${ }^{2}$ What could explain this discrepancy? Prognostic research of patients who are elderly and medically ill consistently highlights poor detection of depression and low rates of treatment, either of which could worsen the outcome.

This view is strengthened by a recent study in which fluoxetine was compared with placebo over 8 weeks in older patients who were medically ill. ${ }^{3}$ For the 42 patients who completed the study (82 entered it), recovery from depression in the actively treated group was approximately double that of those given a placebo. Although there are several flaws in these studies, there is a message: active detection and treatment may substantially improve the outcome. Medical comorbidity makes these patients among the most difficult to treat, but the current range of antidepressant drugs is such that most elderly people with depression, however frail, could be prescribed a drug that they can tolerate.

Robert C Baldwin, DM, FRCP, FRCPsych Central Manchester Health Care NHS Trust Manchester, UK

1 Koenig HG, George LK, Peterson BL, et al. Depression in medically ill hospitalized older adults: prevalence, characteristics, and course of symptoms according to six diagnostic schemes. Am J Psychiatry 1997;154:1376-83.

2 Cole MG. The prognosis of depression in the elderly. CMAJ 1990;143:633-9.

3 Evans M, Hammond M, Wilson K, et al. Placebo-controlled treatment trial of depression in elderly physically ill patients. Int J Geriatr Psychiatry 1997;12:817-24. 